1 Review

\title{
2 The Endocannabinoid System of Animals
}

3 Robert J. Silver ${ }^{1}$

41 Chief Medical Officer, RxVitamins; $r$ silver@drsilverdvm.com

5 Simple Summary: The recent discovery of the Endocannabinoid System, and its ubiquitous 6 presence in nearly all animals, has opened the door to novel approaches targeting pain management, cancer therapeutics, modulation of neurologic disorders, stress reduction, anxiety management and inflammatory diseases. Endocannabinoid-related molecules, both endogenous

9 and exogenous, are able to function as direct ligands or otherwise influence the ECS. This review 10 article introduces the reader to the endocannabinoid system in animals, and documents its 11 potential as a source for emerging therapeutics.

12 Abstract: The endocannabinoid system has been found to be pervasive in mammalian species. It

13 has also been described in invertebrate species primitive as the Hydra. Insects apparently are 14 devoid of this otherwise ubiquitous system that provides homeostatic balance to the nervous and 15 immune systems, as well as many other organ systems. The endocannabinoid system (ECS) has 16 been defined to consist of three parts: 1. Endogenous ligands, 2. G-protein coupled receptors 17 (GPCRs), and 3. Enzymes to degrade and recycle the ligands. Two endogenous molecules have 18 been identified as ligands in the ECS to date. These are the endocannabinoids: Anandamide 19 (arachidonoyl ethanolamide) and 2-AG (2-arachidonoyl glycerol). Two G-coupled protein 20 receptors have been described as part of this system, with other putative GPC being considered. 21 Coincidentally, the phytochemicals produced in large quantities by the Cannabis sativa L plant, and 22 in lesser amounts by other plants, can interact with this system as ligands. These plant-based 23 cannabinoids are termed, phytocannabinoids. The precise determination of the distribution of 24 cannabinoid receptors in animal species is an ongoing project, with the canine cannabinoid 25 receptor distribution currently receiving the most interest in non-human animals. 
26 Keywords: Endocannabinoid system; Anandamide; 2-AG; Cannabis; Cannabinoid Receptor 1;

27 Cannabinoid Receptor 2; PPARS a, b; Ht1a; TRPV1; GPR55; cannabidiol; CBD; THC; CBG; CBC;

28 tetrahydrocannabinol

\section{Introduction}

31 Common to nearly all animals except the Phyla Protozoa and Insecta, the endocannabinoid system arose in the phylogeny concurrently with the development of the nervous system as multicellular animals developed increasing complexity. This system was unknown to scientists until the mid-1990's, but research into this fascinating and clinically useful system is advancing rapidly, especially with the use of state-of- the-art LC-MS analyzers, and immunohistochemical and polymerase chain reaction (PCR) analytic technologies.

For over 70 years scientists have been hobbled by the legal and regulatory prohibitions related to research into cannabis and its associated molecules. Phytocannabinoids from Cannabis sativa L. and the naturally occurring endocannabinoids can serve as ligands in the ECS.

\section{The Endocannabinoid System}

The endocannabinoid system (ECS) has been identified in nearly all animals, from complex mammals like primates to phylogenetically primitive animals such as the cnidarians. The near universal presence and early emergence of the ECS, evolutionarily, is a strong indicator of its biological importance. Cannabinoid receptors are expressed in most animals, including vertebrates (mammals, birds, reptiles, and fish) and invertebrates (sea urchins, leeches, mussels, nematodes, and others). 
51 that the major function of the ECS in this primitive organism is to control its feeding response [1]. It

52 is evident from this data that all veterinary species contain an ECS. Therefore, an understanding of

53 the ECS in these species is critical to the development of clinical applications for endocannabinoids

54 and the phytocannabinoids, terpenes, and flavonoids derived primarily from Cannabis sativa L.

55 The ECS was discovered during the search for the biological targets for the recently described

56 structure of the psychotropic phytocannabinoid, $-\Delta-9$-tetrahydrocannabinol (THC) [2]. THC is the

57 only psychotropic cannabinoid found in Cannabis sativa $\mathrm{L}$ and is responsible for some of its

58 biomedical activity along with the non-psychotropic cannabinoids such as cannabidiol (CBD),

59 Cannabigerol (CBG), Cannabichromene (CBC), other minor cannabinoids, terpenes and

60 flavonoids.

61 Research sponsored by the National Institute on Drug Abuse in Bethesda, MD led to the discovery of the GPCR which was named $\mathrm{CB}_{1}$. Two years following the discovery of the $\mathrm{CB}_{1}$ receptor, Makriyannis characterized a second GPCR named $\mathrm{CB}_{2}$ [3]. Both the $\mathrm{CB}_{1}$ and the $\mathrm{CB}_{2}$ receptors play important roles in many essential biological processes, including neuronal plasticity, pain, anxiety, inflammation in general and especially neuroinflammation, immune function, metabolic regulation, reward, craving and bone growth [4]. quickly identified the endogenous ligands that bind to the cannabinoid receptors (CBR). These endogenous ligands are the endocannabinoids (eCB), arachidonoyl ethanolamide (AEA), a long-chain fatty acid amide, was described in 1992, and was named: "Anandamide" by Mechoulam,

71 after the Sanskrit "Ananda" meaning bliss, as this endogenous molecule is responsible for feelings of well-being. The ester of this fatty acid amide, 2-arachidonoyl glycerol (2-AG), was discovered shortly thereafter in 1995 [5-7]. Both of these compounds are able to activate both $\mathrm{CB}_{1}$ and $\mathrm{CB}_{2}$ receptors, and were found to produce effects similar to THC, which is the only phytocannabinoid that binds orthosterically to the cannabinoid receptors. 

eCBs are produced ad hoc by enzymes within the cell membrane that are activated by calcium ion elevation. The level of endocannabinoids is termed the "Endocannabinoid tone" and that level varies based on the specific tissue in which they are found. These levels are dependent upon their rate of production minus the rate of their enzymatic degradation by another set of enzymes, fatty acid amide hydrolase (FAAH) and monoacyl-glycerol lipase (MAGL). Respectively, these enzymes deactivate AEA and 2-AG.

By definition, the endocannabinoid system consists of these three parts:

1. Endogenous ligands

2. Membrane receptors

3. Deactivating enzymes

The regulation of endocannabinoid tone is modulated by a transport mechanism that carries released eCBs retrograde from the post-synaptic cell membrane to bind with the cannabinoid receptors present on the pre-synaptic membrane, and then carry them back to the post-synaptic neuron for degradation by their respective enzymes, FAAH or MAGL.

Endocannabinoids act on the presynaptic endocannabinoid receptors following their release from the post-synaptic neuronal membrane. The eCBs modulate neurotransmitter release by inhibiting the influx of intracellular calcium which in turn inhibits the release of neurotransmitters. eCBs undergo rapid re-uptake by the cells and are then degraded. Their half-life is quite brief. Following release, they undergo rapid reuptake by the cell and are then degraded. The production of eCBs can be stimulated by injury or excessive neuronal discharges [8]. ECS signaling comes in two

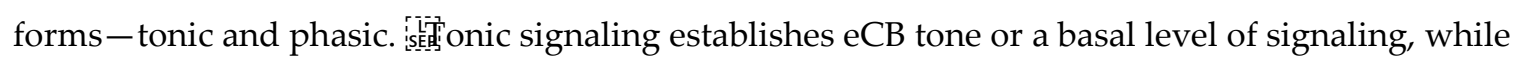
phasic signaling involves changes over time of eCB levels.

Omega-3 fatty acids are essential for the proper regulation sisedf the ECS tone, since polyunsaturated fatty acids feed is irectly into the eCB signaling pathways [9]. These studies into the ECS were able to characterize the two cannabinoid receptors, the proteins that modulate their function and the eCB 
102

103

104

105

106

107

108

109

110

111

112

113

114

115

116

117

118

119

120

121

122

123

124

125

126

127

family of compounds that encompass the ECS system. The ECS plays a major role in the regulation of many aspects of animal physiology. Today we know that the CB1 cannabinoid receptor is the most abundant GPCR in the human brain but is also present in many other organs such as the heart, blood vessels, liver, lungs, and the digestive system, as well as fat and sperm cells [10].

The CB1 receptor belongs to the Class A rhodopsin-like family of GPCRs. It is found primarily in the central nervous system with concentrations found in the cortex, hippocampus, outflow of the basal ganglia, and cerebellum. There can be both intra- and interspecies differences in the anatomical location of cannabinoid receptors in the ECS. It's important to note that CB1 in humans is not prevalent in the brain stem or medulla oblongata, the organs responsible for controlling vital autonomic functions such as breathing and heartbeat. This is a strong contributing factor to the safety profile of cannabinoids in humans and the main reason that it is nearly impossible to overdose on THC [11].

For dogs this is not true. Government studies in the 1970's determined that dogs have a very high density of cannabinoid receptors in the cerebellum and brain stem and medulla oblongata [12]. This concentration of $\mathrm{CB}$ receptors in the cerebellum explains "Static Ataxia", the neurologic reaction to THC that is specific to dogs naive to THC. Static ataxia was first described in the literature by Dixon in 1899 [13]. Cannabinoid receptors are found to a lesser extent in the periphery of cardiovascular, immune, gastrointestinal, and reproductive tissues. CB2 receptors are located primarily in immune cells, among them leukocytes and those of the spleen and tonsils [14]. The CB1 and CB2 receptors share a significant degree of homology despite being located primarily in the CNS and immune system, respectively.

One of the functions of cannabinoid receptors in the immune system is the modulation of cytokine release. Activation of B- and T- cell CB2 receptors by cannabinoids leads to inhibition of adenyl cyclase in these cells and to a reduced response to immune challenge [15]. Both CB1 and CB2 are cause a decrease in adenyl cyclase activity and the cAMP pathway. They also stimulate mitogen-activated protein kinase (MAPK) cascades, modulate ion channels, and modify intracellular 
128 calcium levels and subsequent neurotransmitter release [16-19]. Potassium channel activation can

129 also serve as a signaling mechanism for the CB2 receptor $[20,21]$.

130 Cannabinoid action is not limited to signaling outside of the cell. Fatty acid-binding proteins (FABP)

131 are essential for the transport of cannabinoids into the cell. Once inside the cell they bind to

132 cannabinoid receptors on the outer mitochondrial membrane and have several pathways: 1. Recruit

133 nuclear transcription factors which modify gene expression [22] and 2. Regulate neuronal energy

134 metabolism [23]. CRIP1a, another cannabinoid signaling protein, inhibits constitutive eCB signaling

135 [24]. Mitochondrial CB1 receptors modify cellular respiration through inhibition of soluble adenyl

136 cyclase and reducing activity in the electron transport chain. Mitochondrial receptors may also play

137 a role in the pro-apoptotic mechanisms of cannabinoids upon cancer cells, via the release of

138 ceramide and its role in creating ER stress, leading to autophagy and if the cell cannot correct itself,

139 apoptosis ensues [25].

140 3. Veterinary ECS: Our Current State of Knowledge

141 Most of what we know about the medical and health benefits of cannabis relates to humans and not

142 animals. Many of the biological interactions of the endocannabinoid system occur across most of the

143 animal species. Therefore, describing the medical and health benefits of cannabis as relates to

144 humans, are starting points for evaluating cannabis as a therapeutic agent in veterinary species.

145 The CB1 receptor is highly conserved across all mammalian species, but there are significant

146 primary sequence differences that have been discovered between the human and rat cannabinoid

147 CB2 receptors and the newly cloned canine cannabinoid receptor, CB2. It was found that the binding

148 affinities for canine CB2 receptor were 30 times less than those measured for human and rat CB2

149 receptors. The functional properties of the cannabinoid CB2 receptor are highly dependent upon the

150 level of receptor expression and the nature of the selected signaling pathway [26].

\section{$151 \quad 3.1$ Anatomical Localization of Cannabinoid Receptors in the Dog}


152

153

154

155

156

157

158

159

160

161

162

163

164

165

166

167

168

169

170

171

172

173

175

\subsubsection{Cannabinoid Receptor 1} neurons. Schwann cells in the PNS.

\subsubsection{Canine $\mathrm{CB}_{1}$ Receptor Localization}

Immunohistochemistry was used to anatomically localize the CB1 receptor in the normal canine nervous system. Nervous systems from a healthy 4-week-old puppy, three 6-month-old dogs, and one 10-year-old dog were evaluated. Strong "dot-like immunoreactivity" was found in the neutrophils of the cerebral cortex, cornu ammonis (CA), and dentate gyrus of the hippocampus, midbrain, cerebellum, medulla oblongata, and gray matter of the spinal cord. Dense CB1 expression was found in fibers of the globus pallidus and substantia nigra surrounding immunonegative

Astrocytes were consistently positive in all examined regions. In the PNS, CB1

immunohistochemistry stained neurons and satellite cells of the dorsal root ganglia and myelinating

The younger dog in this study had lower CB1 expression in the brain. The density of receptor expression in human fetal and neonatal brain tissue was greater when compared to the younger dog examined. Lower CB1 expression has been found in aged rats in specific regions, most prominently in the cerebellum, cerebral cortex, and basal ganglia and less prominently in the hippocampus. This reduction in CB1 density with age in these rats was consistent with the findings in the older dog examined in this study [27]. Previous studies have identified CB1 receptors in salivary glands [28], hair follicles [29], skin, and hippocampus in dogs [30].

Immunohistochemistry was used to study the localization of CB1 receptors on developing canine embryo (30 days old) with a commercially available antibody. CB1 receptor immunoreactivity was found primarily in epithelial tissues and included most structures of the central and peripheral nervous system, inner ear, olfactory epithelium and related structures, eye, and thyroid gland [30]. 
176

177

178

179

180

181

182

183

184 Clinically normal dogs have a homogeneous distribution of CB1 and CB2 receptors in all epidermal

185

186

187

188

189

190

191

192

193

194

195

196

197

198

199

200

201
- Cytoplasm of basal and suprabasal layer cells

- Hair follicle inner epithelial root sheaths and arrector pili muscles

- Undifferentiated sebocytes at the periphery of sebaceous glands

- Mast cells and fibroblasts

- Upregulated in atopic dermatitis

\subsubsection{Cannabinoid Receptor 2} layers. This is different than in human epidermis where the CB1 receptor is mainly detected in epidermal spinosum and granulosum layers CB2 is detected mainly in basal keratocytes. Both CB1 and $\mathrm{CB} 2$ receptors have been found in the skin of healthy dogs and dogs with atopic dermatitis. The epidermis of dogs is thinner than that of humans (2-3 nucleated layers in the dog versus 6-7 in the human), which might account for this difference. Hyperplastic epidermal changes were observed in dogs with atopic dermatitis. Strong CB1 and CB2 immunoreactivity was identified in suprabasal keratinocytes. Weak CB1 and strong CB2 immunoreactivity was found in basal keratinocytes indicating upregulation of these receptors during inflammation. CB1 and CB2 agonists decrease mast cell degranulation. To summarize, cannabinoid receptor localization on the skin of the dog was found in the cytoplasm of epidermal and follicular keratinocytes, sweat and sebaceous gland epithelial cells, and the mesenchymal dermal cells [31].

\subsubsection{Canine CB2 Receptor Localization}

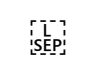

- $\quad$ Epidermis.

- Cytoplasm of cells in the basal and suprabasal layers.

- Hair follicles in the basal and suprabasal cells of the outer and inner epithelial root sheaths. 
- Mild immunoreactivity in cells of arrector pili muscles and secretory and ductal cells of sweat glands.s[sp?

- Sebaceous glands in the cytoplasm and peripheral reserve cells.

- Mast cells, fibroblasts, and endothelial cells.ispep

- Lymph nodes.

\subsection{Invertebrate ECS}

212 The two cannabinoid receptors, CB1 and CB2, have been found in mammals, birds, reptiles, and fish.

213 In a study of seven representative species of invertebrates, McPartland used tritiated ligand binding

214 assays to characterize the cannabinoid receptors in Ciona intestinalis (Deuterostomia), Lumbricus

215 terrestris (Lophotrochozoa), Peripatoides novae-zealandiae (Onychophora), Jasus edwardi (Crustacea),

216 Panagrellus redivivus (Nematoda) [the beer mat nematode], Actinothoe albocincta [white striped

217 anemone] (Cnidaria), and Tethya aurantium (Porifera) [Orange Puffball sponge] [32].

219 Cannabinoid binding was detected in all species studied except for the sea anemone (A. albocincta)

220 and sponge (T. aurantium). The receptors were consistent with CB1 receptors but not CB2 receptors.

221 Three of the organisms tested, earthworm (L. terrestris), velvet worm (P. novae-zealandiae), and mat nematode ( $P$. redivivus), were compared to a standard CB1 ortholog in rat cerebellar tissue. A high affinity binding interaction was observed at various concentrations characteristic of CB1 receptors.

225 The authors of this study hypothesize that cannabinoid receptors evolved in the last common

226 ancestor of bilaterians, with secondary loss in insects and other clades. After conducting a systematic

227 literature review, the authors found that cannabinoid receptors have been identified in sea urchins, 
leeches, earthworms, hydra, lobster (H. americanus and J. edwardi), and the beer mat nematode ( $P$. redivivus), but not the nematode (C. elegans). No binding was observed in sponges (Porifera).

In a separate study, McPartland found that insects (Apis mellifera [western honey bee]), Drosophila melanogaster [common fruit fly], Gerris marginatus [water strider], Spodoptera frugiperda [fall armyworm moth larva], and Zophobas atratus [darkling beetle]) are devoid of cannabinoid receptors.

234 This loss of $\mathrm{CB}$ receptors is unique to comparative neurobiology, in that no other known mammalian 235 neuroreceptor has been found to be missing in insects (Ecdysozoa). The authors suggest that the lack 236 of cannabinoid receptors in insects is due to their lack of ligands, in that insects produce little or no arachidonic acid, the precursor to the biosynthesis of endocannabinoids [33].

\subsection{The Endocannabinoid System and Disease [11]}

Most of the work that has been done to delineate the effect of the endocannabinoid system on various diseases, has focused on the human animal and experimental models with laboratory

243 animals. The assumption can be made that comparable benefits can be achieved in veterinary species 244 via comparable mechanisms of action. Clinical studies are needed in veterinary species to further 245 delineate applications for phytocannabinoids and for endocannabinoid molecules.

\subsubsection{Modulation of Anxiety and Stress}

248 Deficiencies in eCB signaling have been implicated in the etiology of a variety of conditions

249 including PTSD, migraine, and fibromyalgia. Circulating levels of eCBs have been found to be

250 markedly decreased in these disorders. The decline in circulating eCBs is correlated with

251 anxiety-like behaviors. Chronic environmental stress leads to a down-regulation of $\mathrm{CB}_{1}$ receptors

252 combined with reduced levels of AEA but increased levels of 2-AG [34]. 
253 CBD in animal models was shown to have anxiolytic properties. Healthy human subjects who had to

254 perform a stressful public speaking test (SPST), were given a $300 \mathrm{mg}$ dose of CBD isolate which

255 reduced their subjective anxiety comparable to anxiolytic benzodiazepam [35]. CBD has also been

256 shown to ameliorate some of the undesirable effects of THC and when administered concurrently

257 helps to temper the psychoactivity of THC.

\subsubsection{Modulation of Inflammatory Conditions}

Inflammation is a common condition that underlies the development and progression of many diseases and health conditions. The ECS has been shown both in vivo and in vitro to be involved in sclerosis, rheumatoid arthritis, colitis and hepatitis.

268 function of these receptors is to modify the inflammatory response. These receptors protect the host

269 from the pathogenesis of these conditions through the induction of multiple anti-inflammatory

270 pathways such as suppression of T-cell-mediated immune responses. They induce apoptosis of

271 T-cells and suppress pro-inflammatory cytokines and chemokines and also inhibit T-effector cell proliferation at the same times as stimulating proliferation of T-regulatory cells.

274 The cannabis plant contains many molecules that reduce inflammation. THC and CBD both have

275 strong anti-inflammatory properties, while CBC, CBG, and THCV have also demonstrated

276 anti-inflammatory properties. The apoptotic mechanism of phytocannabinoids upon immune cells is

277 to activate of CD95 which induces both Bcl-2 and caspase cascades. Cannabinoids have also been

278 demonstrated to promote the production of anti-inflammatory interleukins such as IL-10 while 
279 inhibiting the production of pro-inflammatory cytokines such as TNF- $\alpha$ in a CB 1 - dependent fashion 280 [36].

282 Non-steroidal anti-inflammatory drugs (NSAIDs) produce their anti-inflammatory response

283 through interactions with the ECS. Acetaminophen is metabolized in the liver resulting in

$284 N$-arachidonoylphenolamine (AM-404) which acts as both a cannabinoid receptor agonist and eCB

285 reuptake inhibitor [37]. These interactions also block the conversion of arachidonic acid into

286 inflammation and pain promoting prostaglandins [38].

Terpenes can also have anti-inflammatory activity. Among the terpenes, $\alpha$-pinene, $\beta$-myrcene, anti-inflammatory effect. $\beta$-caryophyllene is the only terpene known to bind to cannabinoid receptors thus attenuating inflammation in $\mathrm{CB}_{2}$ - receptor dependent fashion [39].

\subsubsection{Modulation of Pain}

Endocannabinoids modulate neural conduction of pain signals by both reducing the nociceptive neural signal of pain, and by reducing inflammation by means of activation of cannabinoid receptors, either by endogenous ligands or $\Delta-9$-THC.

$\mathrm{CB}_{1}$ receptors modulate neurotransmitter release in the brain and spinal cord. $\mathrm{CB}_{1}$ receptors are also

300 present in nociceptive and non-nociceptive sensory neurons of the dorsal root ganglion and

301 trigeminal ganglion, as well as macrophages mast cells and epidermal keratinocytes.

$303 \mathrm{CB}_{2}$ receptors are found in cells of hematopoietic origin. There are few $\mathrm{CB}_{2}$ receptors in the brain,

304 spinal cord, and dorsal root ganglion. $\mathrm{CB}_{2}$ receptors will up-regulate in response to peripheral nerve 
damage. These cannabinoid receptors regulate neuroimmune interactions and interfere with inflammatory hyperalgesia.

The endocannabinoids anandamide and 2-AG are produced in tissue that has been injured, and activate cannabinoid receptors to suppress the sensitization of the nerve to nociceptive signals and/or to suppress inflammation. The anandamide modulates pain by: 1. Inhibiting nociceptive signals at the synapse by activating $\mathrm{CB}_{1}$ receptors; 2 . Transformed by COX-2 enzymes into pain-relieving molecules (prostamides). 3) Reduces inflammation through activating $\mathrm{CB}_{2}$ and other receptors. 2-AG plays a role in the descending modulation of pain during acute stress. Both molecules are produced as the body's first response to tissue injury [40,41].

\subsubsection{Metabolic Effects}

Satiety in part, is modulated through the hypothalamic pro-opiomelanocortin (POMC) neurons. Activation of the $\mathrm{CB}_{1}$ receptor inhibits the POMC neurons and results in appetite increase. This reduction in satiety can be attributed to the inhibitory effects of cannabinoids on the release of the appetite suppressant $\alpha$-melanocyte-stimulating hormone ( $\alpha$-MSH). There is an inverse correlation between levels of orexin-A and $\alpha$-MSH. Orexin A induces hyperphagia by increasing levels of 2-AG [42].

Cannabis users who have hyperexia, also known as "the munchies", eat more calories due to this inhibitory effect of cannabinoids. Paradoxically, users of cannabis are slimmer than non-users of cannabis. The prevalence of obesity is lower in regular cannabis users compared to non-users, even after adjusting for important variables such as age, sex, and tobacco smoking status. [43]. Cannabis users display lower levels of fasting insulin and better insulin sensitivity than their non-using counterparts [44]. These are the metabolic effects of the endocannabinoid system, due in part to the presence of cannabinoid receptors on the mitochondria. 
331 The ECS plays a key role in modulating cell differentiation, cell proliferation, and cell death.

332 Additionally, cannabinoids such as THC and CBD stimulate appetite and reduce the emetic 333 responses seen in chemotherapy. These qualities make the ECS an attractive target for use in cancer 334 therapy. Cannabinoids can also down-regulate certain cancers through modulation of gene 335 expression.

336 In lung cancer the administration of CBD results in an upregulation of the expression of the 337 intracellular adhesion molecules (iCAM) which in turn prevent metastasis [45].

338 In gliomas the administration of CBD results in reduction in the expression of pro-angiogenic factors 339 in a dose-dependent fashion. THC, when administered in conjunction with CBD, has been found to 340 be synergistic to inhibit proliferation and survival of human glioblastoma cells [46].

341 The in vitro addition of CBD to breast cancer cells was found to down regulate the expression of ID-1, 342 a large contributor to metastasis of breast cancer cells. [47].

\section{$343 \quad$ 3.3.6 The Role of Antioxidants and Neuroprotection}

344 Cannabinoids act as antioxidants and neuroprotectants. The US National Institute of Health (NIH)

345 holds a patent on these compounds for this purpose [48]. As antioxidants, cannabinoids can

346 neutralize reactive oxygen species. Cannabinoids inhibit voltage-gated calcium channels resulting in 347 the inhibition of the release of glutamate. This neurotransmitter stimulates neuronal depolarization.

348 Glutamate is released during periods of ischemia and other traumatic brain events. In excess,

349 glutamate itself is toxic and can lead to neuronal cell death through excitotoxic stress [49].

350 Compounds with antioxidant properties are often neuroprotective, for instance through reduction of 351 toxic reactive oxygen species (ROS) produced during ischemic metabolism. Both THC and CBD

352 have been shown to have antioxidant properties [50]. THC and CBD are both able to prevent 
353 glutamate induced neurotoxicity. The neuroprotective effect of these compounds is independent of

354 their CB receptor binding activity. THC and CBD reduce ROS in vitro, similar to known antioxidants 355 such as ascorbate and butylated hydroxytoluene (BHT). CBD has been shown to protect against 356 cerebral ischemic injury [51], and also attenuates Alzheimer's-related neuroinflammation in animal 357 models [52].

358 Terpenes have strong antioxidant properties and can also serve as neuroprotectants. $\beta$-myrcene, a 359 common terpene in high THC cannabis but not low THC cannabis (hemp), protected against 360 oxidative stress and histological damage induced by ischemia-reperfusion in a mouse model of 361 cerebral ischemia. Terpenes in cannabis with antioxidant properties include $\beta$-caryophyllene, 362 limonene, and $\beta$-myrcene [53]. Nearly all of the cannabinoids have been found to have potent 363 antioxidant properties. [54,55].

364 The ECS is involved in the development of many neurodegenerative conditions. Cannabinoids have 365 been shown to have neuroprotective properties, possessing the ability to reduce neuroinflammation, 366 and promote neurogenesis [56-58].

367 In Alzheimer's Disease (AD) cannabinoids are able to clear the toxic beta amyloid (A $\beta)$ plaques 368 associated with this disease. AD has been found to be associated with a loss of the body's natural 369 production of eCBs, which has been defined as a clinical endocannabinoid deficiency syndrome 370 (CEDS), described later in this article.

371 CBD has also been shown to reduce the expression of genes implicated in the phosphorylation of the 372 tau protein the hyperphosphorylation of which leads to the formation of neurofibrillary tangles that

373 further contributes to the progression of the disease [59]. Furthermore, cannabinoids have been

374 demonstrated to enhance the clearance of $\mathrm{A} \beta$ from the brain as well as prevent the inflammatory 375 cascade that is produced by the accumulation of these mis-folded proteins intracellularly [60,61]. 
377 Cannabinoids modulate blood pressure and heart rate, either increasing or decreasing blood

378 pressure and heart rate, depending upon local conditions [62-64].

379 CBD has a direct effects on arteries, causing vaso-dilation, and subsequent hypotension as a result.

380 This is a mild hypotensive effect, but could be problematic in uncompensated cardiac disease. CBD

381 has a protective effect against vascular damage due to hyperglycemia from Type 2 diabetes, diabetic

382 angiopathies and also with systemic inflammatory processes. The antioxidant and

383 anti-inflammatory effects of CBD mediate these cardiovascular effects $[65,66]$.

384 CBD demonstrated anti-arrhythmic effects following coronary artery occlusion in rats. This study

385 found that these anti-arrhythmic effects were mediated through non-receptor pathways that did not

386 involve the $\mathrm{CB}_{1}$ receptor.

$387 \mathrm{CB}_{1}$ antagonists increased blood pressure and left ventricular contractile performance in a group of

388 rats bred to have spontaneous hypertension. Reductions in blood pressure, cardiac contractility and

389 vascular resistance were mediated via the inhibitory effects of CBD on the enzyme FAAH by

390 increasing the serum half life of anandamide in normotensive rats. $\mathrm{CB}_{1}$ antagonists inhibited these

391 effects. $\mathrm{CB}_{1}$ antagonists lowered blood pressure better in the hypertensive rats versus normotensive

392 rats. This may be due to the upregulation of $\mathrm{CB}_{1}$ receptors in heart and aortic endothelium in

393 hypertensive rats, but not the normotensive cohort [67].

\subsubsection{Modulation of Pulmonary Function}

Inhaled and oral THC can create bronchodilation up to two hours following administration. $\mathrm{CB}_{1}$ receptor activation inhibits cholinergic contraction, which allows it to inhibit bronchospasms. This

may be why asthma sufferers find some comfort from cannabis use [68]. CBD decreased pulmonary

398 inflammation and improved pulmonary function tests in murine models of inflammatory lung

399 disease, as well as improving pulmonary function in a model of COPD [69]. THC resulted in a

400 reduction of allergen-induced mucus production [70]. 


\subsubsection{Clinical Endocannabinoid Deficiency Syndrome (CEDS)}

402

403

404

405

406

407

408

409

410

411

412

413

Russo has postulated that several chronic conditions may be due to deficiencies in eCB signaling. PTSD, migraine, IBS, PTSD and fibromyalgia are of particular note in this Clinical Endocannabinoid Deficiency Syndrome [71,72]. There are known mutations in ECS genes that contribute to such deficiencies, which helps to explain the genetic component of these diseases. Other causes that can affect the constitutive tone, or systemic level of eCBs include certain pharmaceuticals and diseases that can deplete $\mathrm{eCB}$ levels or interfere with $\mathrm{eCB}$ production. Human patients with mutations in CNR1 and DAGLA genes show signs of CEDS [73]. Human IBS patients with mutations in the CNR1 gene, were found to also have altered rates of colonic transit [74]. With PTSD, fear extinction is impaired in patients who were homozygous for a CNR1 mutation [75].

These disorders all have in common markedly decreased systemic levels of eCB. Circulating eCB deficiencies are also inversely correlated with anxiety-like behaviors. Environmental stressors when chronic will down-regulate $\mathrm{CB}_{1}$ receptors and reduce levels of both AEA and 2-AG [76].

\subsubsection{Problems associated with the Endocannabinoid System}

The psychoactive effects of THC, which is a CBR agonist, are undesirable in veterinary species. Dogs in particular will suffer from Static Ataxia upon exposure to THC at doses $>0.5 \mathrm{mg} / \mathrm{kg}$ IV [13]. As a result, states with medical or adult use marijuana laws, have found an increase in Animal ER admissions for THC toxicosis [77]. A report of the summary of calls to the Pet Poison Hotline found a relative good safety profile for $\mathrm{CBD}$, and THC when given in moderate amounts [78]. Oral tolerance to THC can be achieved in the dog following 7-10 days of a low, sub-psychotropic of $0.05-0.1 \mathrm{mg} / \mathrm{kg}$ BID PO. Concurrent use of CBD in equal or greater amounts than the THC will assist in this process [12]. ECS stimulation that is excessive and prolonged can create memory deficits in humans. Upon cessation of prolonged ECS stimulation withdrawal symptoms will develop. 
425 Two university studies, published in 2018 found CBD, when present in a full-spectrum hemp oil extract, to be safe when given at a high dose of $10 \mathrm{mg} / \mathrm{kg} / \mathrm{day}$ for 6 weeks to Beagles, with only a mild and transient elevation in serum alkaline phosphatase, diarrhea, and no interference with anti-epileptic drugs. As CBD is metabolized via the P450 enzyme system, there is concern that the concurrent use of CBD with drugs that are also metabolized through that pathway may have their pharmacokinetics altered, which could alter their therapeutic value for a given patient [79]. were seen in the 16 client owned dogs with osteoarthritis who were recruited for this study [80]. Lower doses of $0.5 \mathrm{mg} / \mathrm{kg}$ BID have anecdotally been found to be effective for most dogs with

434 osteoarthritis. The second efficacy study evaluated the ability of cannabidiol to reduce the number of 435 breakthrough seizures in dogs with refractory epilepsy. At $2.5 \mathrm{mg} / \mathrm{kg}$ BID, the study found only a partial success for that pilot work. A larger study, funded by the AKC Canine Health Foundation is now being conducted using $4.5 \mathrm{mg} / \mathrm{kg}$ BID as the test dosage to evaluate whether it will be better able to extinguish these breakthrough seizures in these dogs [81].

\subsection{Discussion}

From the research presented in this paper, its obvious that the endocannabinoid system in not just present in nearly all animals, but plays an integral role in maintaining homeostasis for a number of organ systems. The endocannabinoid system modulates the nervous and immune systems and other organ systems through a complex system of receptors and chemical signaling molecules to relieve pain and inflammation, modulate metabolism and neurologic function, promote healthy digestive processes, support reproductive function and embryologic development.

448 The future looks bright as cannabinoid research, in the post-cannabis prohibition era, is finally able to search for additional discoveries regarding the role the endocannabinoid system plays in the 


\subsection{REFERENCES}

453

1. De Petrocellis L, Melck D, Bisogno T et al (1999) Finding of the endocannabinoid signaling system in Hydra, a very primitive organism: possible role in the feeding response. Neuroscience 92:377-387

2. Gaoni $Y$, Mechoulam R (1964) Isolation, structure, and partial synthesis of an active constituent of hashish. J Am Chem Soc 86:1646-1647

3. Makriyannis A (2014) 2012 Division of medicinal chemistry award address. Trekking the cannabinoid road: a personal perspective. J Med Chem 57:3891-3911: 46:101-122:s[s:

5. Devane WA, Hanus L, Breuer A et al (1992) Isolation and structure of a brain constituent that binds to the cannabinoid receptor. Science 258:1946-1949 2-monoglyceride, present in canine gut, that binds to cannabinoid receptors. Biochem Pharmacol 50:83-90

8. Basavarajappa BS (2007) Neuropharmacology of the endocannabinoid signaling system-molecular mechanisms, biological actions and synaptic plasticity. Curr Neuropharmacol 5:81-97 
10. Mackie K (2008) Cannabinoid receptors: where they are and what they do. J Neuroendocrinol 20:10-14:sep?

11. Hartsel JA, Boyar K, Pham A, Silver RJ and Makriyannis A. Cannabis in Veterinary Medicine: Cannabinoid Therapies for Animals; in Nutraceuticals in Veterinary Medicine, eds. Gupta RC et al. (2019) pp121-155. https://doi.org/10.1007/978-3-030-04624-8_10

12. Herkenham M, Lynn AB, Little MD, Johnson MR, Melvin LS, de costa BR, Rice KC. Cannabinoid receptor localization in brain. Proc Natl Acad Sci USA Vol87, Table 1, p 1933, March 1990.

13. Dixon WE. The Pharmacology of Cannabis indica. The British Medical Journal; 2,1354-1357; 1899

14. Pertwee RG (2001) Cannabinoid receptors and pain. Prog Neurobiol 63:569-611

15. Condie R, Herring A, Koh WS et al (1996) Cannabinoid inhibition of adenylate cyclase-mediated signal transduction and interleukin 2 (IL-2) expression in the murine T-cell line, EL4. IL-2. J Biol Chem 271:13175-13183

16. Howlett A, Fleming R (1984) Cannabinoid inhibition of adenylate cyclase. Pharmacology of the response in neuroblastoma cell membranes. Mol Pharmacol 26:532-538:

17. Howlett AC (2002) The cannabinoid receptors. Prostaglandins Other Lipid Mediat 68:619631 iscip:

18. Marcu JP, Schechter JB (2016) Molecular pharmacology of CB1 and CB2 cannabinoid receptors. In: Neuropathology of Drug Addictions and Substance Misuse. Elsevier, London, pp 713-721:

19. Pertwee RG (2005) Pharmacological actions of cannabinoids. In: Cannabinoids. Springer, Cham, pp 1-51 antagonist, SR144528: further evidence for cannabinoid CB2 receptor absence in the rat central nervous system. Eur J Pharmacol 377:117-125 
21. Ho B, Uezono Y, Takada S et al (1999) Coupling of the expressed cannabinoid CB1 and CB2 receptors to phospholipase $\mathrm{C}$ and $\mathrm{G}$ protein-coupled inwardly rectifying $\mathrm{K}+$ channels. Receptors Channels 6:363-374

22. Elmes MW, Kaczocha M, Berger ST et al (2015) Fatty acid-binding proteins (FABPs) are intracellular carriers for $\Delta$ 9-tetrahydrocannabinol (THC) and cannabidiol (CBD). J Biol Chem 290:8711-8721

23. Bénard G, Massa F, Puente N et al (2012) Mitochondrial CB 1 receptors regulate neuronal energy metabolism. Nat Neurosci 15:558-564

24. Smith TH, Blume LC, Straiker A et al (2015) Cannabinoid receptor-interacting protein 1a modulates CB1 receptor signaling and regulation. Mol Pharmacol 87(4):747-765

25. Salazar M, Carracedo A, Salanueva IJ et al. Cannabinoid action induces autophagy-mediated cell death through stimulation of ER stress in human glioma cells. J Clin Invest 119:1359-1372 (2009)

26. Ndong C, O'donnell D, Ahmad S et al (2011) Cloning and pharmacological characterization of the dog cannabinoid CB2 receptor. Eur J Pharmacol 669:24-31

27. Freundt-Revilla J, Kegler K, Baumgärtner W et al (2017) Spatial distribution of cannabinoid receptor type 1 (CB1) in normal canine central and peripheral nervous system. PLoS One 12:e0181064

28. Dall'Aglio C, Mercati F, Pascucci L et al (2010) Immunohistochemical localization of CB1 receptor in canine salivary glands. Vet Res Commun 34:9-12

29. Campora L, Miraqliotta V, Ricci E et al (2012) Cannabinoid receptor type 1 and 2 expression in the skin of healthy dogs and dogs with atopic dermatitis. Am J Vet Res 73:988-995

30. Mercati F, Dall'Aglio C, Pascucci L et al (2012) Identification of cannabinoid type 1 receptor in dog hair follicles. Acta Histochem 114:68-71

31. Pirone A, Lenzi C, Coli A et al (2015) Preferential epithelial expression of type-1 cannabinoid receptor (CB1R) in the developing canine embryo. Springerplus 4:804 
32. McPartland JM, Agraval J, Glesson D et al (2006) Cannabinoid receptors in invertebrates. J Evol Biol 19:366-373

33. McPartland J, Marzo VD, Petrocellis LD et al (2001) Cannabinoid receptors are absent in insects. J Comp Neurol 436:423-429

34. Morena M, Patel S, Bains JS et al (2016) Neurobiological interactions between stress and the endocannabinoid system. Neuropsychopharmacology 41:80

35. Zuardi AW, Cosme RA, Graeff FG et al (1993) Effects of ipsapirone and cannabidiol on human experimental anxiety. J Psychopharmacol 7 (1 Suppl):82-88

36. Klein TW, Lane B, Newton CA, Friedman H (2000) The cannabinoid system and cytokine network. Proc Soc Exp Biol Med 225:1-8 prevented by the blockade of cannabinoid CB1 receptors. Eur J Pharmacol 531:280-281

38. Saliba SW, Marcotequi AR, Fortwängler E et al (2017) AM404, paracetamol metabolite, prevents prostaglandin synthesis in activated microglia by inhibiting COX activity. J Neuroinflammation 14:246

39. Klauke A-L, Racz I, Pradier B et al (2014) The cannabinoid CB2 receptor-selective phytocannabinoid beta-caryophyllene exerts analgesic effects in mouse models of inflammatory and neuropathic pain. Eur Neuropsychopharmacol 24:608-620 Cannabis and Cannabinoid Research Volume 2.1, 2017; doi: 10.1 089/can.2017.0017.

41. Morello G, Imperatore R, Palomba L et al (2016) Orexin-A represses satiety-inducing POMC neurons and contributes to obesity via stimulation of endocannabinoid signaling. Proc Natl Acad Sci USA 113:4759-4764

42. Le Foll B, Trigo JM, Sharkey KA et al (2013) Cannabis and $\Delta 9$ - tetrahydrocannabinol (THC) for weight loss? Med Hypotheses 80:564-567is:sp? 
553 43. Muniyappa R, Sable S, Ouwekerk R et al (2013) Metabolic effects of chronic cannabis 554 smoking. Diabetes Care 36:2415-2422

555 44. Haustein M, Ramer R, Linnebacher M et al (2014) Cannabinoids increase lung cancer cell lysis by lymphokine-activated killer cells via upregulation of ICAM-1. Biochem Pharmacol $92: 312-325$

45. Vaccani A, Massi P, Colombo A et al (2005) Cannabidiol inhibits human glioma cell migration through a cannabinoid receptor-independent mechanism. Br J Pharmacol 144:1032-1036

46. McAllister SD, Christian RT, Horowitz MP et al (2007) Cannabidiol as a novel inhibitor of Id-1 gene expression in aggressive breast cancer cells. Mol Cancer Ther 6:2921-2927:

47. Hampson AJ, Axelrod J, Grimaldi M (2003) Cannabinoids as antioxidants and neuroprotectants. United States Patent \#: 6630507.

48. Hampson A, Grimaldi M, Axelrod J et al (1998) Cannabidiol and (๑) 95:8268-8273

49. Borrelli F, Fasolino I, Romano B et al (2013) Beneficial effect of the non-psychotropic plant cannabinoid cannabigerol on experimental inflammatory bowel disease. Biochem Pharmacol 85(9):1306-1316

50. Hayakawa K et al (2008) Cannabidiol prevents a post-ischemic injury progressively induced by cerebral ischemia via a high-mobility group box1-inhibiting mechanism. Neuropharmacology 55:1280-1286. inhibits $\beta$-amyloid-induced tau protein hyperphosphorylation through $W n t / \beta$-catenin pathway rescue in PC12 cells. J Mol Med 84:253-258 
52. Ciftci O, Ozdemir I, Tanyildizi S et al (2011) Antioxidative effects of curcumin, $\beta$-myrcene and 1,8-cineole against 2, 3, 7, 8-tetrachlorodibenzo-p-dioxin-induced oxidative stress in rats liver. Toxicol Ind Health 27:447-453

53. Calleja MA, Vieites JM, Montero-Meterdez T et al (2013) The antioxidant effect of $\beta$-caryophyllene protects rat liver from carbon tetrachloride-induced fibrosis by inhibiting hepatic stellate cell activation. Br J Nutr 109:394-401

54. Borrelli F, Fasolino I, Romano B et al (2013) Beneficial effect of the non-psychotropic plant cannabinoid cannabigerol on experimental inflammatory bowel disease. Biochem Pharmacol 85(9):1306-1316

55. Jiang W, Zhang Y, Xiao L et al (2005) Cannabinoids promote embryonic and adult hippocampus neurogenesis and produce anxiolytic and antidepressant-like effects. J Clin Invest 115:3104-3116

56. Saito VM, Rezende RM, Teixeira AL (2012) Cannabinoid modulation of neuroinflammatory disorders. Curr Neuropharmacol 10:159-166

57. Van der Stelt M, Veldhuis W, Bär P et al (2001) Neuroprotection by $\Delta 9$-tetrahydrocannabinol, the main active compound in marijuana, against ouabain-induced in vivo excitotoxicity. J Neurosci 21:6475-6479:[SEPp]

58. Esposito G, De Filippis D, Carnuccio R et al (2006) The marijuana component cannabidiol

59. Eubanks LM, Rogers CJ, Beuscher AE IV et al (2006) A molecular link between the active component of marijuana and Alzheimer's disease pathology. Mol Pharm 3:773-777 
602

603

604

605

606

607

608

609

610

611

612

613

614

615

616

617

618

619

620

621

624

625

626

62. Niederhoffer N, Szabo B (1999) Effect of the cannabinoid receptor agonist WIN55212-2 on sympathetic cardiovascular regulation. Br J Pharmacol 126(2):457-466

63. Niederhoffer N, Szabo B (2000) Cannabinoids cause central sympathoexcitation and bradycardia in rabbits. J Pharmacol Exp Ther 294(2):707-713

64. Stanley CP, Hind WH, O'Sullivan SE (2013) Is the cardiovascular system a therapeutic target for cannabidiol? Br J Clin Pharmacol 75:313-322

65. Hepburn C, Walsh S, Wainwright C (2011) 17 Cannabidiol as an anti- arrhythmic, the role of the CB1 receptors. Heart 97:e8

66. Bátkai S et al (2004) Endocannabinoids acting at cannabinoid-1 receptors regulate cardiovascular function in hypertension. Circulation 110:1996-2002

67. Tashkin DP, Shapiro BJ, Frank IM (1974) Acute effects of smoked marijuana and oral $\Delta$ 9-tetrahydrocannabinol on specific airway conductance in asthmatic subjects. Am Rev Respir Dis 109:420-428

68. Ribeiro A et al (2015) Cannabidiol improves lung function and inflammation in mice submitted to LPS-induced acute lung injury. Immunopharmacol Immunotoxicol 37:35-41.

69. Reddy AT, Lakshmi SP, Reddy RC (2012) Murine model of allergen induced asthma. J Visual Exp, JoVE

70. Russo EB (2016a) Beyond cannabis: plants and the endocannabinoid system. Trends Pharmacol Sci 37:594-605

71. Russo EB (2016b) Clinical endocannabinoid deficiency reconsidered: Current research supports the theory in migraine, fibromyalgia, irritable bowel, and other treatment-resistant syndromes. Cannabis Cannabinoid Res 1:154-165

72. Smith DR, Stanley C, Foss $\mathrm{T}$ et al (2017) Rare genetic variants in the endocannabinoid system genes CNR1 and DAGLA are associated with neurological phenotypes in humans. PLoS One 12:e0187926 
73. Camilleri M, Kolar GJ, Vazquez-Roque MI et al (2013) Cannabinoid receptor 1 gene and irritable bowel syndrome: phenotype and quantitative traits. Am J Physiol Gastrointest Liver Physiol 304(5): G553-G560

74. Heitland I, Klumpers F, Oosting RS et al (2012) Failure to extinguish fear and genetic variability in the human cannabinoid receptor 1. Transl Psychiatry 2:e162

75. Hill MN, McLaughlin RJ, Morrish AC et al (2009) Suppression of amygdalar endocannabinoid signaling by stress contributes to activation of the hypothalamicpituitary-adrenal axis. Neuropsychopharmacology 34:2733 marijuana toxicosis in dogs living in a state with legalized medical marijuana: 125 dogs (2005-2010) J Vet Emerg Crit Care 2012;22(6):690-696. Doi: 10.1111/j.1476-4431.2012.00818.x Cannabidiol in Dogs and Cats. Vet Clin Small Anim 48 (2018) 1087-1102. https://doi.org/10.1016/j.cvsm.2018.07.008 Associated with the Administration of Cannabidiol in Healthy Dogs. J AHVMA Vol 52, Fall, 2018 pp34-38. Efficacy of Cannabidiol Treatment in Osteoarthritic Dogs. Front. Vet. Sci. 5:165, doi:10.3389/fvets.2018.00165. AVMA Conference 2018. 
652 My gratitude to Jean Dodds, DVM for inviting me to participate in this Animal.com issue, and to my

653 colleagues, Joshua Hartsel, Andrew Pham, and Kyle Boyar with whom I co-authored the chapter on

654 Cannabinoid Therapies for Animals (11) in the recently published textbook on Nutraceuticals in

655 Veterinary Medicine by Springer Publications. 